\title{
Approximate solution to the speed of spreading viruses
}

\author{
Vicente Ortega-Cejas, ${ }^{1, *}$ Joaquim Fort, ${ }^{2, \dagger}$ Vicenç Méndez, ${ }^{3, \ddagger}$ and Daniel Campos ${ }^{1}$ \\ ${ }^{1}$ Grup de Física Estadística, Departamento de Física, Universitat Autònoma de Barcelona, 08193 Bellaterrra, Spain \\ ${ }^{2}$ Departamento de Física, Universitat de Girona, Campus de Montilivi, 17071 Girona, Catalonia, Spain \\ ${ }^{3}$ Departamento de Medicina, Facultat de Ciències de la Salut, Universitat Internacional de Catalunya, Gomera s/n, \\ 08190 Sant Cugat del Vallès, Barcelona, Spain
}

(Received 20 November 2003; published 24 March 2004)

Recently, it has been shown that the speed of virus infections can be explained by time-delayed reactiondiffusion [J. Fort and V. Méndez, Phys. Rev. Lett. 89, 178101 (2002)], but no analytical solutions were found. Here we derive formulas for the front speed, valid in appropriate limits. We also integrate numerically the evolution equations of the system. There is good agreement with both numerical and experimental speeds.

DOI: 10.1103/PhysRevE.69.031909

PACS number(s): 87.18.Hf, 87.10.+e

\section{INTRODUCTION}

The role of the delay time in the spread of viruses in a plaque has been recently analyzed [1] by considering a delay time for virus diffusion. It has been shown that the delay time plays a crucial role in the dynamics of the advancing virus front, because it substantially reduces the value for the predicted speed as compared to the classical, parabolic model [2]. In this sense, $\tau$ is the time that a virus particle spends, from the moment it is adsorbed into a host bacterium, to take control of it, replicate its proper genetic material, reproduce, and kill the cell. We consider a model of three species, the virus particles $V$, the host bacteria $B$, and the infected host bacteria $I$. There are two reactions involved in the virus expansion over the bacterial colony: (i) the adsorption process, during which a virus particle couples to a host bacterium through its membrane and the cell becomes infected and (ii) the lysis process, at the end of which the cell is killed and the virus progeny outbreak takes place. Thereafter, (iii) the phages disperse and (iv) they infect new hosts, so the process begins again. Let $k_{1}$ be the rate constant of adsorption. The virus particle introduces its genetic material in the infected bacteria and begins the reproduction. After a certain delay time $\tau$ (latent or lag time), the virus particle is completely reproduced and the infected bacterium dies (lysis).

In this work we obtain an analytic expression for the speed of the growth of virus plaques and compare them with the numerical solution of the complete system and with the experimental data. Comparison with the classical or nondelay time models $[2,3]$ are not included because it was already done in [1].

\section{THE MODEL}

\section{A. Virus spreading dynamics}

The process of infection, virus replication, and bacterium death can be summarized by a three species reaction as follows:

\footnotetext{
*Email address: vicente.ortega@uab.es

${ }^{\dagger}$ Email address: joaquim.fort@udg.es

*Email address: vmendez@csc.unica.edu
}

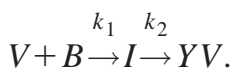

Parameter $Y$ (the "yield") is the production of new viruses per infected bacterium and $k_{2}$ is the rate constant of lysis of infected bacteria.

In order to find a good quantitative agreement with the experimental observations, it has been previously shown [1] that a better way to model the virus diffusion process is by taking into account the delay time between virus adsorption and bacteria death and the spreading of the newborn viruses. In practice, it implies that parabolic or classical reactiondiffusion equation must be replaced by its hyperbolic generalization [4,5], where the mentioned delay time appears explicitly. Assuming logistic dynamics for the growth process, the equations for our models are

$$
\begin{aligned}
\frac{\tau}{2}[V]_{t t}+[V]_{t}= & D_{e f f}[V]_{r r}-k_{1}\left\{[V][B]+\frac{\tau}{2}([V][B])_{t}\right\} \\
& +Y k_{2}\left\{[I]\left(1-\frac{[I]}{[I]_{\max }}\right)\right. \\
& \left.+\frac{\tau}{2}\left[[I]\left(1-\frac{[I]}{[I]_{\max }}\right)\right]_{t}\right\} \\
& {[B]_{t}=-k_{1}[V][B], } \\
{[I]_{t}=} & k_{1}[V][B]-k_{2}[I]\left(1-\frac{[I]}{[I]_{\max }}\right)
\end{aligned}
$$

In these equations $[\cdots]$ denotes concentration and subindices $[\cdots]_{t t},[\cdots]_{t}$, and $[\cdots]_{r r}$ stand for second time derivative, time derivative, and second spatial derivative in the radial direction from the plaque center, respectively. In Eq. (2) $D_{e f f}$ appears instead of the usual diffusion coefficient $D$. The reason is that the diffusing particles, i.e., viruses, do not move in a homogeneous continuous medium (agar in our case) but in the presence of a suspension of ellipsoids (host bacteria) which adsorb them. This is known as hindered diffusion, and the effective diffusion coefficient $D_{\text {eff }}$ for this type of diffusion is related to the usual one, $D$, according to Fricke's equation [6]: 


$$
D_{e f f}=\frac{1-f}{1+\frac{f}{x}} D,
$$

where $f=B_{0} / B_{\max }$ is the ratio of bacteria concentration to its maximum possible value and $x$ takes care of the bacterium shape.

Equations (2)-(4) can be written in terms of dimensionless variables $\bar{B} \equiv[B] / B_{0}, \bar{V} \equiv[V] / B_{0}, \bar{I} \equiv[I] / B_{0}, \bar{t} \equiv k_{2} t$, and $\bar{r} \equiv r \sqrt{k_{2} / D_{e f f}}$ and dimensionless parameters $\bar{\tau} \equiv k_{2} \tau$ and $\kappa \equiv k_{1} B_{0} / k_{2}$, where $B_{0}$ is the initial bacterium concentration. We look for solutions depending only on new variable $\bar{z} \equiv \bar{r}$ $-\bar{c} \bar{t}$ where $\bar{c}>0$ is the dimensionless wave front speed, which is related to dimensional speed $c$ by $\bar{c} \equiv c / \sqrt{D_{e f f} k_{2}}$. As usual, we linearize our equations around the unstable steady state $([V],[B],[I])=\left(0, B_{0}, 0\right), \quad$ i.e., $\quad(\bar{V}, \bar{B}, \bar{I})=\left(\varepsilon_{V}, 1\right.$ $\left.-\varepsilon_{B}, \varepsilon_{I}\right)$, where $\varepsilon \equiv\left(\varepsilon_{V}, \varepsilon_{B}, \varepsilon_{I}\right) \ll 1$. Then solutions to the linearized version of Eqs.(2-4) are given by $\varepsilon \sim \exp (-\lambda \bar{z})$ where, in order to avoid trivial solutions, the following characteristic equation must be satisfied:

$$
\begin{gathered}
\lambda^{3}+\frac{-1+(1+\beta \delta) \bar{c}^{2}}{\left(\beta \bar{c}^{2}-1\right) \bar{c}} \lambda^{2}+\frac{\kappa(1-\beta \gamma)+1}{\beta \bar{c}^{2}-1} \lambda \\
-\frac{\kappa \gamma}{\left(\beta \bar{c}^{2}-1\right) \bar{c}}=0 .
\end{gathered}
$$

For simplicity, we have introduced the parameters $\beta$ $\equiv \bar{\tau} / 2, \gamma \equiv Y-1$, and $\delta \equiv k+1$.

\section{B. Wave front speed}

In order to avoid nonpositive values for concentrations, we must impose that the three solutions for $\lambda$ in Eq. (6) are real, so it must be satisfied that

$$
-4 C_{1}^{3} C_{3}+C_{1}^{2} C_{2}^{2}+18 C_{1} C_{2} C_{3}-4 C_{2}^{3}-27 C_{3}^{2} \geqslant 0,
$$

where $C_{1}, C_{2}$, and $C_{3}$ are the coefficients of second, first, and zeroth powers of $\lambda$, respectively. We rewrite condition (7) in terms of $\xi \equiv \bar{c}^{2}$ and then we get

$$
a_{3} \xi^{3}+a_{2} \xi^{2}+a_{1} \xi+a_{0} \geqslant 0,
$$

where coefficients $a_{i}$ are given by

$$
\begin{gathered}
a_{0}=-4 \gamma \kappa \\
a_{1}=12 \gamma(1+\beta \delta) \kappa-27 \gamma^{2} \kappa^{2}+18 \gamma \kappa[-1+(-1+\beta \gamma) \kappa] \\
+[-1+(-1+\beta \gamma) \kappa]^{2} \\
a_{2}=-12 \gamma(1+\beta \delta)^{2} \kappa+54 \beta \gamma^{2} \kappa^{2} \\
-18 \beta \gamma \kappa[-1+(-1+\beta \gamma) \kappa] \\
-18 \gamma(1+\beta \delta) \kappa[-1+(-1+\beta \gamma) \kappa] \\
-2(1+\beta \delta)[-1+(-1+\beta \gamma) \kappa]^{2} \\
-4[-1+(-1+\beta \gamma) \kappa]^{3},
\end{gathered}
$$

$$
\begin{aligned}
a_{3}= & 4 \gamma(1+\beta \delta)^{3} \kappa-27 \beta^{2} \gamma^{2} \kappa^{2} \\
& +18 \beta \gamma(1+\beta \delta) \kappa[-1+(-1+\beta \gamma) \kappa] \\
& +(1+\beta \delta)^{2}[-1+(-1+\beta \gamma) \kappa]^{2} \\
& +4 \beta[-1+(-1+\beta \gamma) \kappa]^{3} .
\end{aligned}
$$

The speed of the wave front can be calculated numerically from $\bar{c}=\min _{\lambda>0}[\bar{c}(\lambda)]$, where $\bar{c}(\lambda)$ is given by Eq. (6) as it is done in Ref. [1], but now we shall try to obtain an approximated analytical expression for this minimum speed although, for this purpose, we shall make some approximations. On one hand, we define $\epsilon \equiv k_{1} / k_{2} B_{\max }$ which implies that $\kappa=\epsilon f$. As we shall see in detail in the following section, when typical experimental values for the parameters are used, one observes that $\epsilon$ is always a small parameter, i.e., $\epsilon \ll 1$. This fact allows us to expand the coefficients $a_{i}$ up to first order in $\epsilon$, so we get

$$
\begin{gathered}
a_{0}=-4 f \gamma \epsilon, \\
a_{1}=1+2 f[1+\gamma(-3+5 \beta)] \epsilon+O\left(\epsilon^{2}\right), \\
a_{2}=2(1-\beta)+2 f\left[4-3 \beta+\gamma\left(3+2 \beta-4 \beta^{2}\right)\right] \epsilon+O\left(\epsilon^{2}\right), \\
a_{3}=(1-\beta)^{2}+2 f(-1+\beta)\left[-1+2 \beta+\gamma\left(-2+2 \beta+\beta^{2}\right)\right] \epsilon \\
+O\left(\epsilon^{2}\right) .
\end{gathered}
$$

Moreover, if $\beta \gg 1$ is verified, we can simplify Eqs. (10) even further to get

$$
\begin{gathered}
a_{0}=-4 f \gamma \epsilon \equiv r_{0}, \\
a_{1} \simeq 1+10 f \gamma \beta \epsilon \equiv r_{1}, \\
a_{2} \simeq-2 \beta-8 f \gamma \beta^{2} \epsilon \equiv r_{2}, \\
a_{3} \simeq \beta^{2}+2 f \gamma \beta^{3} \epsilon \equiv r_{3} .
\end{gathered}
$$

Then Eq. (8) is reduced to

$$
r_{3} \xi^{3}+r_{2} \xi^{2}+r_{1} \xi+r_{0} \geqslant 0,
$$

where coefficients $r_{i}$ are defined in Eq. (11). The condition critical to the propagation speed is given by Eq. (12) when equality holds, and then it is easy to show that positive solutions for the speed are

$$
\bar{c}_{1}=2 \sqrt{\frac{f \gamma \epsilon}{1+2 f \beta \gamma \epsilon}}, \quad \bar{c}_{2}=\sqrt{\frac{1}{\beta}},
$$

or, in terms of the dimensional variables,

$$
\begin{gathered}
c_{1}=2 \sqrt{D \frac{1-f}{1+f / x} \frac{k_{1} B_{\max }(Y-1) f}{1+\tau k_{1} B_{\max }(Y-1) f}}, \\
c_{2}=\sqrt{\frac{2 D}{\tau} \frac{1-f}{1+f / x}} .
\end{gathered}
$$

According to the principle of marginal stability $[7,8]$, from both expressions for the wave front speed, we must choose the minimal one. This will be confirmed in Sec. III 


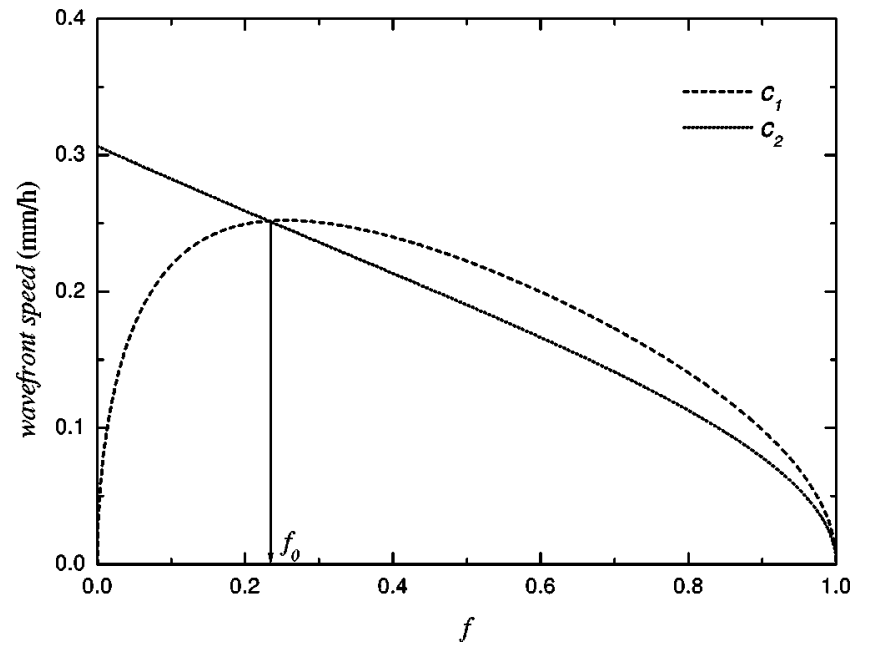

FIG. 1. Solutions to Eq. (12) when equality holds as functions of the bacterial relative concentration $f$. The selected value for the speed is the minimal one, i.e., $c_{1}$ if $f<f_{0}$ and $c_{2}$ if $f>f_{0}$. Both functions are drawn for $B_{\max }=10^{7} \mathrm{ml}^{-1}, k_{1}=0.7 \times 10^{-9} \mathrm{ml} / \mathrm{min}, k_{2}$ $=1.39 \mathrm{~min}^{-1}, \tau=18.4 \mathrm{~min}$, and $Y=34.5$.

below by means of numerical integrations of Eqs. (2)-(4). It is easy to show the existence of a critical value of $f$, namely, $f_{0}$, such that $c_{1}<c_{2}$ if $f<f_{0}$ and $c_{1}>c_{2}$ if $f>f_{0}$. Figure 1 shows both $c_{1}$ and $c_{2}$ as functions of $f$ for typical experimental values of parameters. Then we can write the minimal speed as follows:

$$
c_{\min }= \begin{cases}2 \sqrt{D \frac{1-f}{1+\frac{f}{x}} \frac{f}{\tau\left(f+f_{0}\right)}} & \text { if } 0 \leqslant f \leqslant f_{0} \\ \sqrt{\frac{2 D}{\tau} \frac{1-f}{1+\frac{f}{x}}} & \text { if } f_{0} \leqslant f \leqslant 1,\end{cases}
$$

where $f_{0}$ is defined as

$$
f_{0} \equiv\left[\tau k_{1} B_{\max }(Y-1)\right]^{-1} .
$$

\section{COMPARISON TO OBSERVATIONS}

We compare in this section the results of Eq. (15) with the experimental values for virus $\mathrm{T} 7$ which spread in a medium containing agar-immobilized E. coli bacteria. We also compare the new results with numerical integrations performed on system (2)-(4). The values of the parameters are: $B_{\max }$ $=10^{7}-10^{8} \mathrm{ml}^{-1}, \quad k_{1}=(1.29 \pm 0.59) \times 10^{-9} \mathrm{ml} / \mathrm{min}, \quad k_{2}$ $=1.39 \mathrm{~min}^{-1}, \quad \tau=18.4 \mathrm{~min}, \quad Y=34.5, \quad D=4 \times 10^{-8} \mathrm{~cm}^{2} / \mathrm{s}$, and $x=1.67$. To obtain Eq. (15) we have assumed that $\beta$ $\gg 1$ (in fact $\beta=k_{2} \tau / 2=12.8$ ) which basically implies that delay time is large enough, so comparison to nondelay time modes are out of place. The other assumption is $\epsilon \ll 1$ and from the experimental data we have that $\epsilon$ ranges from 5 $\times 10^{-3}$ to 0.135 .

In Fig. 2(a) we have taken $B_{\max }=10^{7} \mathrm{ml}^{-1}$ and the two extreme values for $k_{1}$. We plot the analytic solution for the
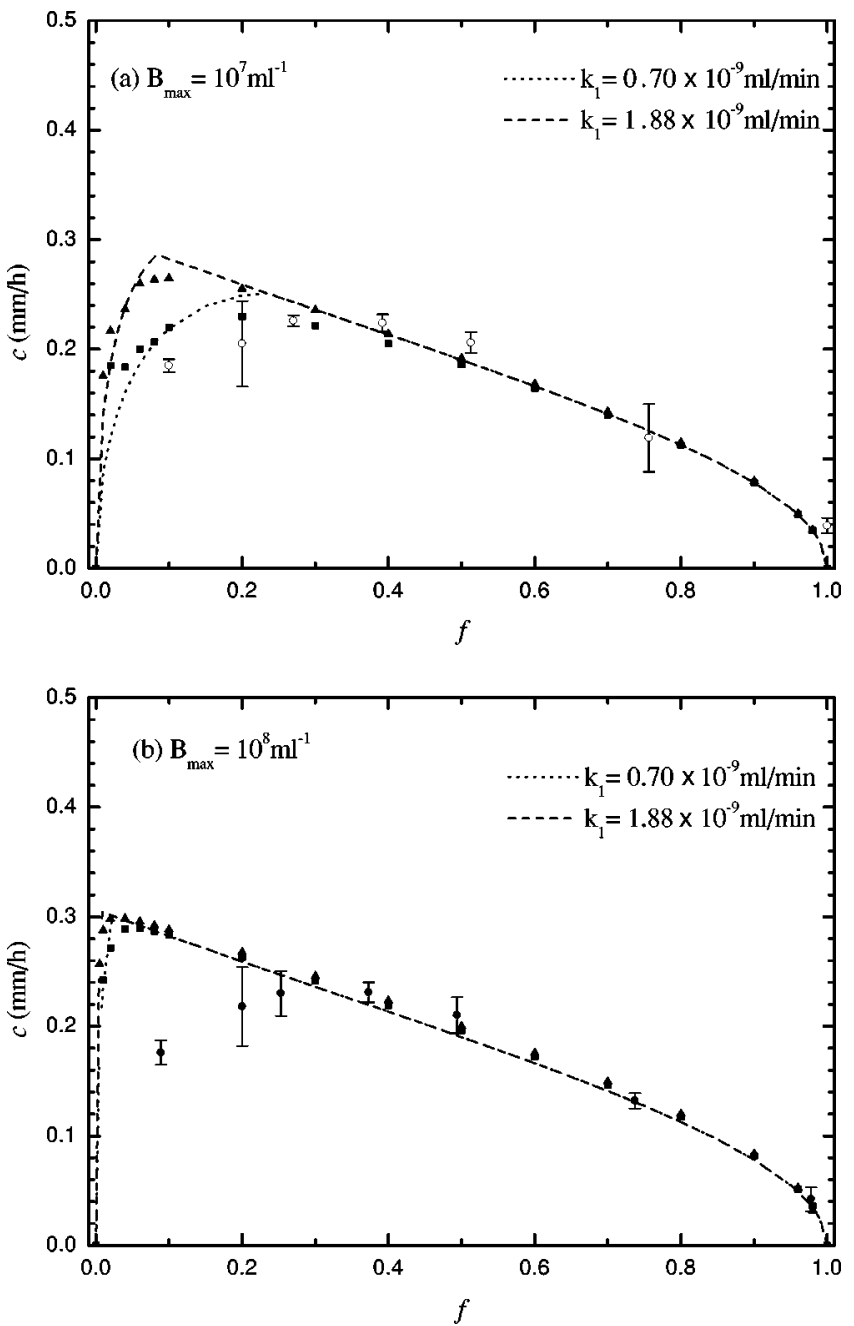

FIG. 2. Curves: speed of the growth of T7 virus plaques on E. coli as a function of the bacterial relative concentration according to expression (15). Symbols: squares and triangles, numerical integrations of Eqs. (2)-(4); open and closed circles, experimental data. In (a) $\epsilon=5 \times 10^{-3}$ for the dotted line and square symbols and $\epsilon=0.013$ for the dashed line and triangles. In (b) $\epsilon=0.05$ for the dotted line and square symbols and $\epsilon=0.13$ for the dashed line and triangles. For all cases $\beta=12.8$.

speed of the front (15) (lines) and the results from numerical solutions of the system (2)-(4) (symbols) and observe good agreement with the experimental results. In Fig. 2(b) we take $B_{\text {max }}=10^{7} \mathrm{ml}^{-1}$ for the same values of $k_{1}$ as before where good agreement with experimental results is also found.

\section{DISCUSSION}

In the present paper we have found an explicit expression for the speed of the growth of virus plaques (15) which is valid only if the parameter values satisfy the specified conditions, i.e., $\epsilon \ll 1$ and $\beta \gg 1$. Moreover, we have performed numerical integrations on Eqs. (2)-(4) in order to compare their results with predictions from Eq. (15). We can see this comparison in Fig. 2 and we note that both approaches are in 
good quantitative agreement, especially when the bacterial relative concentration $f$ is far from the value $f_{0}$. We also include in Fig. 2 experimental data to realize the validity of the time-delayed diffusion-reaction models to explain the wave front speed of these phenomena.

\section{ACKNOWLEDGMENTS}

This research was partially supported by the Ministerio de Ciencia y Tecnología (Spain) through Grant Nos. BFM 20000351-C03-01 and SGR 2001-00186.
[1] J. Fort and V. Méndez, Phys. Rev. Lett. 89, 178101 (2002).

[2] J. Yin and J.S. McCaskill, Biophys. J. 61, 1540 (1992).

[3] L. You and J. Yin, J. Theor. Biol. 200, 365 (1999).

[4] D. Jou, J. Casas-Vázquez, and G. Lebon, Extended Irreversible Thermodynamics (Springer-Verlag, Berlin, 1996).
[5] J. Fort and V. Méndez, Phys. Rev. Lett. 82, 867 (1999).

[6] H. Fricke, Phys. Rev. 24, 575 (1924).

[7] G. Dee and J.S. Lager, Phys. Rev. Lett. 50, 383 (1978).

[8] W. van Saarloos, Phys. Rev. A 37, 211 (1998). 